\title{
Disturbed spontaneous brain activity pattern in patients with primary angle-closure glaucoma using amplitude of low-frequency fluctuation: a fMRI study
}

This article was published in the following Dove Press journal:

Neuropsychiatric Disease and Treatment

29 July 2015

Number of times this article has been viewed

\author{
Xin Huang ${ }^{1, *}$ \\ Yu-Lin Zhongl,* \\ Xian-Jun Zeng ${ }^{2}$ \\ Fuqing Zhou ${ }^{2}$ \\ Xin-Hua Liu' \\ Pei-Hong Hu' \\ Chong-Gang Pei ${ }^{1}$ \\ Yi Shao' \\ $\mathrm{Xi-Jian} \mathrm{Dai}^{2}$ \\ 'Department of Ophthalmology, \\ ${ }^{2}$ Department of Radiology, The First \\ Affiliated Hospital of Nanchang \\ University, Nangchang, Jiangxi, People's \\ Republic of China \\ *These authors contributed equally \\ to this work
}

Objective: The aim of this study is to use amplitude of low-frequency fluctuation (ALFF) as a method to explore the local features of spontaneous brain activity in patients with primary angle closure glaucoma (PACG) and ALFFs relationship with the behavioral performances.

Methods: A total of twenty one patients with PACG (eight males and 13 females), and twenty one healthy subjects (nine males and twelve females) closely matched in age, sex, and education, each underwent a resting-state functional magnetic resonance imaging scan. The ALFF method was used to assess the local features of spontaneous brain activity. The correlation analysis was used to explore the relationships between the observed mean ALFF signal values of the different areas in PACG patients and the thickness of the retinal nerve fiber layer (RNFL).

Results: Compared with the healthy subjects, patients with PACG had significant lower ALFF areas in the left precentral gyrus, bilateral middle frontal gyrus, bilateral superior frontal gyrus, right precuneus, and right angular gyrus, and higher areas in the right precentral gyrus. In the PACG group, there were significant negative correlations between the mean ALFF signal value of the right middle frontal gyrus and the left mean RNFL thickness ( $r=-0.487, P=0.033$ ), and between the mean ALFF signal value of the left middle frontal gyrus and the right mean RNFL thickness ( $r=-0.504, P=0.020)$.

Conclusion: PACG mainly involved in the dysfunction in the frontal lobe, which may reflect the underlying pathologic mechanism of PACG.

Keywords: angle-closure glaucoma, amplitude of low-frequency fluctuation, functional magnetic resonance imaging, resting state, spontaneous activity, retinal nerve fiber layer

\section{Introduction}

Primary angle-closure glaucoma (PACG), a type of optic neuropathy, is characterized by progressive loss of retinal ganglion cells with degenerative optic neuropathy. Glaucoma is the most common cause in the world resulting in irreversible blindness. ${ }^{1}$ Angle-closure glaucoma affects Asian and Eskimo eyes more frequently than in any other race with similar predisposing dimensions. ${ }^{2}$ Several risk factors have been identified for PACG, including sex, age, shallow anterior chamber depth, and most importantly (and the only modifiable risk factor for glaucoma) elevated intraocular pressure (IOP). Therefore, reducing IOP could prevent glaucoma from developing. However, some patients still lose vision in spite of normal IOP after glaucoma surgery. Therefore, it has been found that in addition to the retinal ganglion cells, glaucoma also damages post-retinal mechanisms, which include the lateral geniculate nucleus (LGN) of the thalamus, and the primary visual cortex. Neuronal degeneration of the LGN and activity changes in the primary visual cortex, have been discovered using
Correspondence: Chong-Gang Pei; Yi Shao

Department of Ophthalmology, The First Affiliated Hospital of Nanchang University, No 17, YongWai Zheng Street, DongHu District, Nanchang 330006, Jiangxi, People's Republic of China

Tel +8679188692520

Email peichongang@sina.com; freebee99@163.com
Neuropsychiatric Disease and Treatment 2015:1/ 1877-1883

1877 
experimental models of glaucoma in primates. ${ }^{3-5}$ Although glaucoma is commonly considered as a retinal disease, there is substantial evidence that not only the retina ganglion cells and the optic nerve but also the upstream components of the visual pathway and visual cortex, are affected ${ }^{6}$ However, The mechanisms of optic neuropathy and cerebral functional area damage caused by glaucoma remain to be further explored.

Diffusion tensor imaging can quantitatively measure the anterior visual pathway compression using the fractional anisotropy (FA) and mean diffusivity, and has been applied to glaucoma using various post-processing techniques. ${ }^{7}$ A previous study found glaucoma patients had an altered FA and mean diffusivity measurements in the optical disk, optic chiasma, and optic radiation, and strong correlations were found between the stage of glaucoma and these parameters. ${ }^{8}$ The glaucoma was involved with the optic disk, optic nerve, primary visual processing, and lateral geniculate body, and showed a positive correlation with the degree of glaucoma. ${ }^{4,9,10}$ Other evidence demonstrated the relationship between the degree of visual field loss and the altered blood flow of the visual cortex, or the blood perfusion of primary visual cortex. ${ }^{11}$ Although these findings showed neuronal morphological changes in the glaucoma, there was far less evidence for the neuromechanism changes.

It is proposed that the resting-state functional magnetic resonance imaging (fMRI), a field of research that is currently receiving a lot of interest in neuroimaging and one that is suitable for central mechanism research, can detect the spontaneous neuronal activity of the human brain and can provide new insights into the pathophysiology of disease, because of its advantage in not requiring exposure to radioactive tracers, accurate positioning, and combining functional imaging with structural imaging. ${ }^{12}$ The amplitude of lowfrequency fluctuation (ALFF) is an index in which the square root of the power spectrum is integrated in a low-frequency range, to detect the regional spontaneous neuronal activity in blood oxygenation level dependent (BOLD) signal. ALFF investigates the low-frequency BOLD signal fluctuation from the aspect of regional brain activity during rest, reflecting intensity of regional spontaneous brain activity. Previous studies have demonstrated that the ALFF showed good to moderate test-retest reliability. ${ }^{12,13}$ The simple calculation and reliable characterization of the ALFF measurement makes it a suitable and useful tool for resting-state fMRI data analysis in order to investigate a disease trait. The ALFF measurement has been investigated as being a reliable biomarker for many neurological conditions and could provide some important information for understanding several diseases such as epilepsy, ${ }^{14}$ Parkinson, ${ }^{15}$ sleep deprivation, ${ }^{12}$ and obstructive sleep apnea. ${ }^{16}$ Our study is the first to use the ALFF method to investigate the regional spontaneous brain activity in PACG and its relationship with the mean retinal nerve fiber layer thickness (RNFLT).

\section{Materials and methods Subjects}

Twenty one patients with PACG (13 females and eight males) were recruited from the Department of Ophthalmology at The First Affiliated Hospital of Nanchang University. All patients met the following criteria: 1) the IOP from two measurements were both higher than $21 \mathrm{mmHg}(1 \mathrm{mmHg}=0.133 \mathrm{kPa}) ; 2)$ shallow anterior chamber and narrow corner signs were found in the slit lamp and gonioscopy exams; 3 ) a change of typical glaucoma optic disk cup-to-disc ratio (CDR) $>0.6$ in funduscopic examination, also, the patient had visual field damage, including the central scotoma, nasal side of the ladder, bow scotoma, annular scotoma, tubular vision, or temporal side guide; 4) no other ocular diseases (iridocyclitis and ocular trauma), or optic neuropathy (such as infection, inflammation, ischemic diseases, and compressibility damage), history of ocular operation, intraocular diseases, and other diseases that affect the visual field (such as pituitary damage, demyelinating disease, human immunodeficiency virus, diabetic retinopathy, and age-related macular degeneration); 5) no obvious abnormality in the brain parenchyma through head magnetic resonance imaging (MRI); 6) right-handedness; 7) no history of inborn or acquired diseases such as psychiatric disorder, hypertension, diabetes mellitus, coronary artery disease, and no addictions such as to heroin, smoking, or alcohol; 8) no foreign implants in the body; and 9) moderate body shape and weight (Body Mass Index is between $18.5 \mathrm{~kg} / \mathrm{m}^{2}$ and $24.9 \mathrm{~kg} / \mathrm{m}^{2}$ ).

Twenty one healthy subjects (HSs) (twelve females and nine males) who were similar in age, sex, and education to the twenty one patients with PACG, were recruited for this study. All HSs met the following criteria: 1) no abnormalities in visual pathways and brain parenchyma through head MRI; 2) no ocular disease, and naked eye corrected visual acuity (VA) $>1.0 ; 3$ ) a normal nervous and mental system, no hemicrania; and 4) no contraindications for MRI.

This study was approved by The Human Research Ethics Committee of the First Affiliated Hospital of Nanchang University. All subjects participated voluntarily and were informed of the purpose, methods, and the potential risks; all subjects signed an informed consent form.

The cumulative clinical measurement, such as mean RNFLT (using the CIRRUSTM HD-OCT), CDR, and IOP, was calculated as the sum of the measurements in both eyes of each participant. 


\section{MRI parameters}

MRI scanning was performed on a 3-Tesla MRI scanner (Siemens, Erlangen, Germany). High-resolution T1-weighted images were acquired with a three-dimensional spoiled gradient-recalled sequence in an axial orientation: 176 images (repetition time $=1,900 \mathrm{~ms}$, echo time $=2.26 \mathrm{~ms}$, thickness $=1.0 \mathrm{~mm}$, gap $=0.5 \mathrm{~mm}$, acquisition matrix $=256 \times 256$, field of view $=250 \mathrm{~mm} \times 250 \mathrm{~mm}$, flip angle $=9^{\circ}$ ) were obtained. Finally, 240 functional images (repetition time $=2,000 \mathrm{~ms}$, echo time $=30 \mathrm{~ms}$, thickness $=4.0 \mathrm{~mm}$, gap $=1.2 \mathrm{~mm}$, acquisition matrix $=64 \times 64$, flip angle $=90^{\circ}$, field of view $=220 \mathrm{~mm} \times 220 \mathrm{~mm}, 29$ axial slices with gradient-recalled echo-planar imaging pulse sequence), covering the entire brain, were obtained.

\section{fMRI data analysis}

Functional data were checked by MRIcro software (www.MRIcro.com) to exclude defective data. The first ten time points of the functional images were discarded due to the possible instability in the initial MRI signal and the participants' adaptation to the scanning environment. On the basis of MATLAB 2010a (MathWorks, Natick, MA, USA), the rest of the data preprocessing was performed with DPARSFA (http://rfmri.org/DPARSF) software, including Digital Imaging and Communications in Medicine form transformation, slice timing, head motion correction, spatial normalization, and smooth with a Gaussian kernel of $6 \times 6 \times 6 \mathrm{~mm}^{3}$ full-width at halfmaximum. Motion time courses were obtained by estimating the values for translation $(\mathrm{mm})$ and rotation (degrees) for each subject. Participants who had more than $1.5 \mathrm{~mm}$ maximum displacement in $\mathrm{x}, \mathrm{y}$, or $\mathrm{z}$ and $1.5^{\circ}$ of angular motion during the entire fMRI scan would be rejected. The Friston six head motion parameters were used to regress out head motion effects based on recent work showing that higher-order models were more effective in removing head motion effects. ${ }^{17,18}$ Linear regression was also applied to remove other sources of spurious covariates along with their temporal derivatives, including the signal from a ventricular region of interest (ROI), and the signal from a region centered in the white matter. ${ }^{19}$ Of note, the global signal was not regressed out in the present data as was in our previous studies, ${ }^{12,16,20}$ due to controversy around removing the global signal in the preprocessing of resting-state data. ${ }^{21,22}$ After the head motion correction, the fMRI images were spatially normalized to the Montreal Neurological Institute space criteria using the standard echo-planar imaging template and resampling the images at a resolution of $3 \mathrm{~mm} \times 3 \mathrm{~mm} \times 3 \mathrm{~mm}$. After preprocessing, the time series for each voxel were linearly detrended to reduce low-frequency drift, physiological high frequency respiratory, cardiac noise, and time series linear detrending. To reduce the global effects of variability across the participants, the ALFF of each voxel was divided by the global mean ALFF value for each participant.

\section{Brain-behavior correlation analysis}

Based on the ALFF findings, the different brain regions between groups were classified as ROIs with REST software (http:// www.resting-fmri.Sourceforge.net). For each ROI, the mean ALFF value was extracted by averaging the ALFF value over all voxels. Finally, correlation analysis was performed to investigate the relationship between the mean ALFF value in each of those different areas in the PACG group and the related behavioral performances.

\section{Statistical analysis}

Subject characteristics, including age, weight, height, course of PACG , and other findings of visual testing parameters, were tested using Student's $t$-tests to compare the PACG patients with the HSs using IBM Statistical Pack-age for the Social Sciences version 19.0 (SPSS 19.0), and a $P$-value $<0.05$ was deemed significant. The chi-square test was used for categorical data. For functional data, two-sample $t$-test was used to analyze the difference between the two groups, with age and sex as nuisance covariates of no interest. A corrected significance level of individual voxel $P<0.005$ and a cluster size of 26, using an AlphaSim-corrected cluster threshold of $P<0.05$, was used to determine statistical significance.

\section{Results \\ Behavioral results}

There were no significant differences in age $(P=0.08)$, weight ( $P=0.303)$, and height $(P=0.265)$ between the $P A C G$ patients and the HSs. Significant differences were seen in the best-corrected VA-right $(P=0.015)$, best-corrected VA-left $(P<0.05)$, RNFLT-right $(P=0.01)$, and RNFLT-left $(P<0.001)$, between the PACG patients and the HSs (Table 1).

\section{ALFF differences}

Compared with HSs, PACG patients had significantly lower ALFF areas in the left precentral gyrus, bilateral middle frontal gyrus, bilateral superior frontal gyrus, right precuneus, and right angular gyrus, and higher ALFF area in the right precentral gyrus (Figure 1 and Table 2).

\section{Correlation analysis}

In the PACG group, there were negative correlations between mean ALFF value of the right middle frontal gyrus and the left mean RNFLT ( $r=-0.468 ; P=0.033$ ), between the mean ALFF value of the left middle frontal gyrus and the right RNFLT 
Table I Demographic characteristics in PACG subjects and HSs

\begin{tabular}{|c|c|c|c|}
\hline & PACG subjects & HSs & $P$-value \\
\hline Sex (male/female) & $8 / 13$ & $9 / 12$ & 0.753 \\
\hline Age (years) & $51.95 \pm 13.80$ & $60.57 \pm 4.61$ & 0.08 \\
\hline Weight (kg) & $56.95 \pm 5.4 I$ & $58.67 \pm 5.24$ & 0.303 \\
\hline Height $(\mathrm{cm})$ & $159 \pm 8.54$ & $|62.8| \pm 7.8 \mid$ & 0.265 \\
\hline Course of PACG (days) & $77.67 \pm 49.31$ & N/A & N/A \\
\hline Best-corrected VA-right & $0.42 \pm 0.38$ & $1.26 \pm 0.26$ & $0.015^{*}$ \\
\hline Best-corrected VA-left & $0.52 \pm 0.44$ & $1.36 \pm 0.30$ & $0.03 *$ \\
\hline Best-corrected VA-PACG & $0.22 \pm 0.32$ & $\mathrm{~N} / \mathrm{A}$ & N/A \\
\hline RNFLT-right $(\mu \mathrm{m})$ & $75.90 \pm 17.03$ & $130.24 \pm 10.57$ & $0.01 *$ \\
\hline RNFLT-left $(\mu \mathrm{m})$ & $80.43 \pm 24.03$ & $135.43 \pm 7.50$ & $<0.00 \mathrm{I}^{\#}$ \\
\hline RNFLT-PACG & $68.24 \pm 16.57$ & $\mathrm{~N} / \mathrm{A}$ & $\mathrm{N} / \mathrm{A}$ \\
\hline
\end{tabular}

Notes: Significant at $* P<0.05$; $P<0.001$, using a chi-square test for sex data and an independent $t$-test for continuous data. $P$-value is between PACG and HSs. Data presented as mean $\pm S D$.

Abbreviations: PACG, primary angle-closure glaucoma; HSs, healthy subjects; N/A, not applicable; VA, visual acuity; RNFLT, retinal nerve fiber layer thickness.

( $r=-0.504 ; P=0.020$ ), and between the right mean RNFLT and the course of the PACG ( $r=-0.495 ; P=0.022)$, (Figure 2$)$.

\section{Discussion}

During the resting state, correlated spontaneous fluctuations occur within spatially distinct and functionally related groups of cortical and subcortical regions, consisting of the human brain's intrinsic functional networks. ${ }^{23}$ Altered features during the resting state can serve as a marker to promote further understanding of the possible pathogenesis of multiple diseases such as primary insomnia, ${ }^{20}$ obstructive sleep apnea, ${ }^{16,24}$ and sleep deprivation. ${ }^{25}$ Therefore, it is critical to take the intensity of regional spontaneous brain activity in the task-free state into account to better understand how the brain functions adapt in PACG.
As well as the resting-state functional connectivity, the ALFF is an important aspect of the resting-state fMRI study and may provide further ways to understand the PACG-related functional reorganization. Our previous studies have suggested that the ALFF method may be a reliable and robust marker to examine the brain activity between groups. ${ }^{12,16,26}$ In the present study, it was found that PACG showed lower ALFF areas in the left precentral gyrus, bilateral middle frontal gyrus, bilateral superior frontal gyrus, right precuneus, right angular gyrus, and a higher ALFF area in the right precentral gyrus. Furthermore, negative correlations were found between the mean ALFF value of the right middle frontal gyrus and the left mean thickness of the RNFL, and between the mean ALFF value of the left middle frontal gyrus and the right RNFLT, which may suggest that the ALFF analysis is an early biomarker for detecting the altered brain activity and could be useful for indexing the clinical features.

The precentral gyrus is a part of the primary motor cortex that contains large neurons, which connect to the muscle. ${ }^{27}$ Song et $\mathrm{al}^{28}$ found that primary open-angle glaucoma (POAG) patients showed decreased regional homogeneity areas in the bilateral precentral gyrus and bilateral postcentral gyrus. Dai et $\mathrm{al}^{29}$ demonstrated that POAG patients showed a significant decrease in functional connectivity between the right precentral gyrus and primary visual cortex (BA17). In support of these findings, we found PACG patients showed lower ALFF area in the left precentral gyrus and higher ALFF area in the right precentral gyrus, which may suggest a harmful effect on the precentral gyrus.

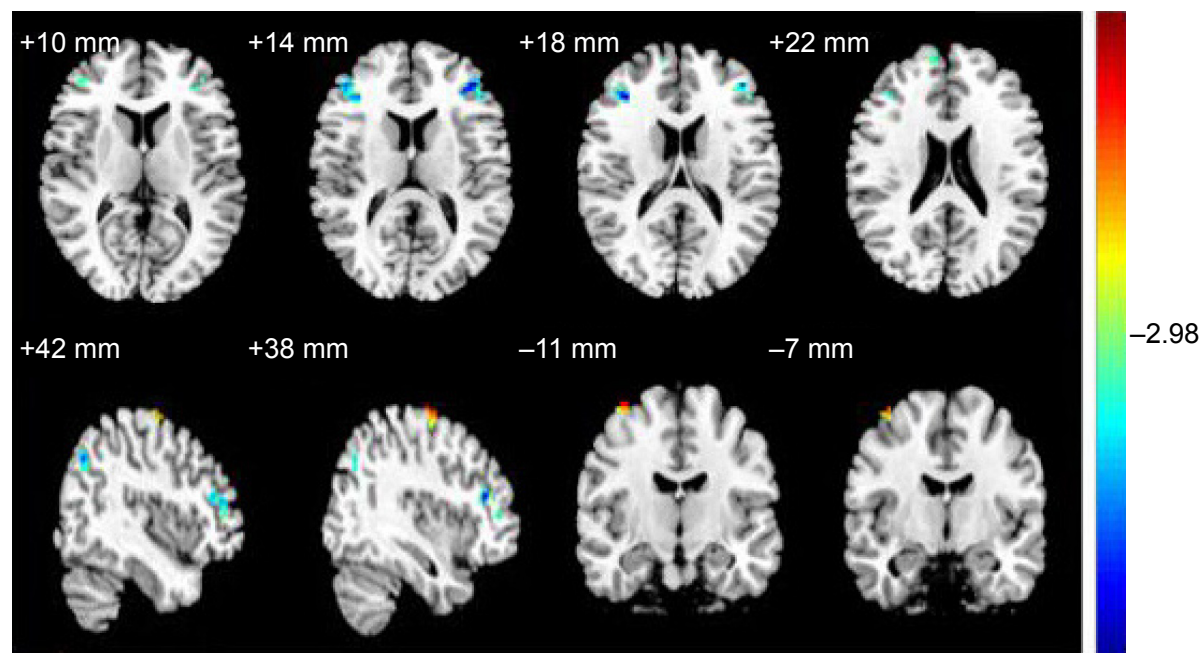

Figure I The different ALFF areas between the PACG subjects and HSs.

Notes: The different brain regions are seen in the bilateral precentral gyrus, bilateral middle frontal gyrus, bilateral superior frontal gyrus, right precuneus and right angular gyrus. The red areas denote higher ALFF brain regions, and the blue areas denote lower ALFF brain regions.

Abbreviations: ALFF, amplitude of low-frequency fluctuation; PACG, primary angle-closure glaucoma; HSs, healthy subjects. 
Table 2 Brain regions with significant differences in ALFF between HSs and PACG subjects

\begin{tabular}{|c|c|c|c|c|c|c|c|}
\hline \multirow[t]{2}{*}{ Condition } & \multirow[t]{2}{*}{ Brain areas } & \multirow[t]{2}{*}{ BA } & \multirow[t]{2}{*}{$t$-score of peak voxel } & \multirow[t]{2}{*}{ Voxels } & \multicolumn{3}{|c|}{ MNI coordinates } \\
\hline & & & & & $\mathbf{x}$ & $y$ & $\mathbf{z}$ \\
\hline $\mathrm{PACG}<\mathrm{HSs}$ & Right middle frontal gyrus & 10 & -4.6243 & 48 & 36 & 33 & 18 \\
\hline PACG $<$ HSs & Left middle frontal gyrus & 10 & -4.8234 & 39 & -42 & 42 & 15 \\
\hline $\mathrm{PACG}<\mathrm{HSs}$ & Right superior frontal gyrus & 9 & -3.9157 & 27 & 6 & 57 & 27 \\
\hline PACG $<$ HSs & Right precuneus & 23 & -4.8576 & 30 & 9 & -54 & 30 \\
\hline PACG $<$ HSs & Left precentral gyrus & 9 & -4.9299 & 34 & -45 & 12 & 33 \\
\hline PACG $<$ HSs & Right angular gyrus & 39 & -4.3023 & 51 & 45 & -66 & 33 \\
\hline PACG $<$ HSs & Left superior frontal gyrus & 9 & -4.3136 & 43 & -18 & 24 & 39 \\
\hline PACG $>$ HSs & Right precentral gyrus & 6 & 4.418 & 26 & 26 & -12 & 69 \\
\hline
\end{tabular}

Notes: The statistical threshold was set at voxel with $P<0.005$, cluster size with $P<0.05$ and cluster size $\geq 26$ between PACG and HSs, corrected by AlphaSim.

Abbreviations: ALFF, amplitude of low-frequency fluctuation; HSs, healthy subjects; PACG, primary angle-closure glaucoma; BA, Brodmann area; MNI, Montreal Neurological Institute.
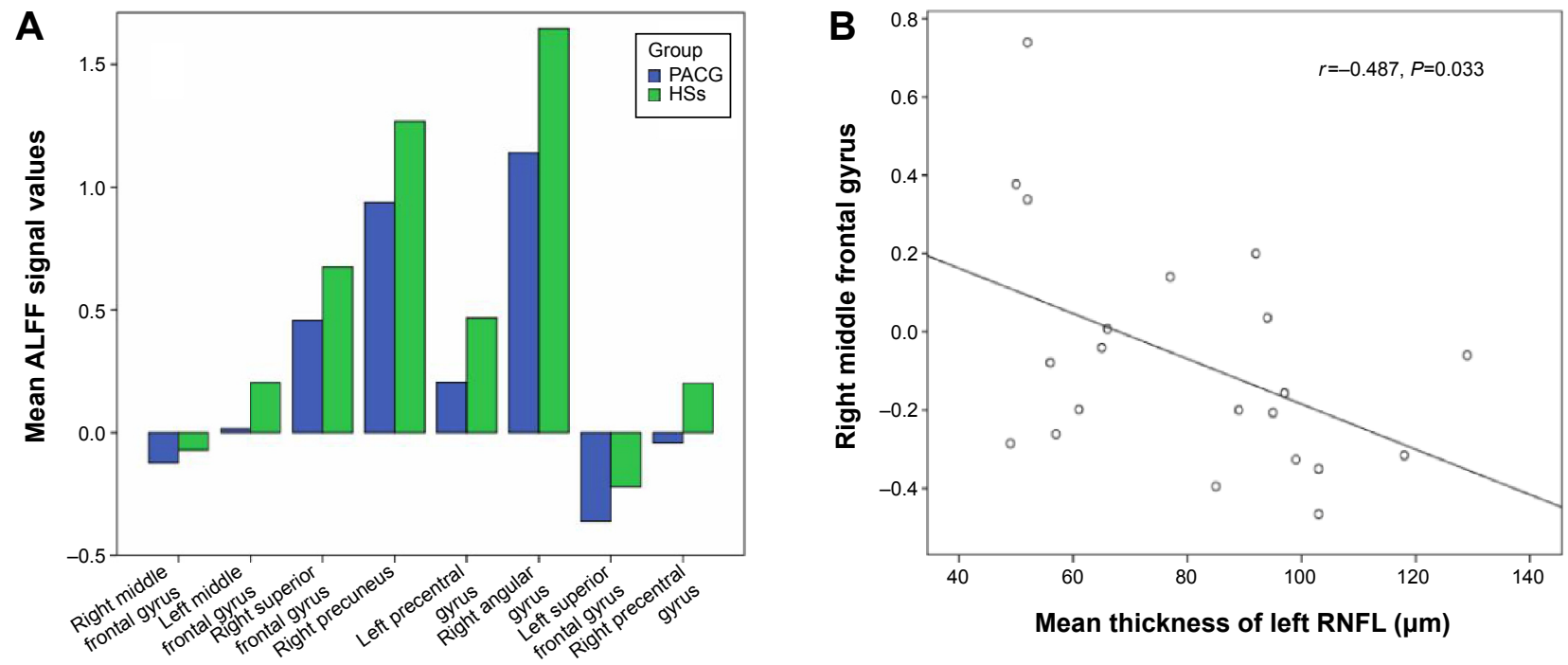

Altered ALFF regions
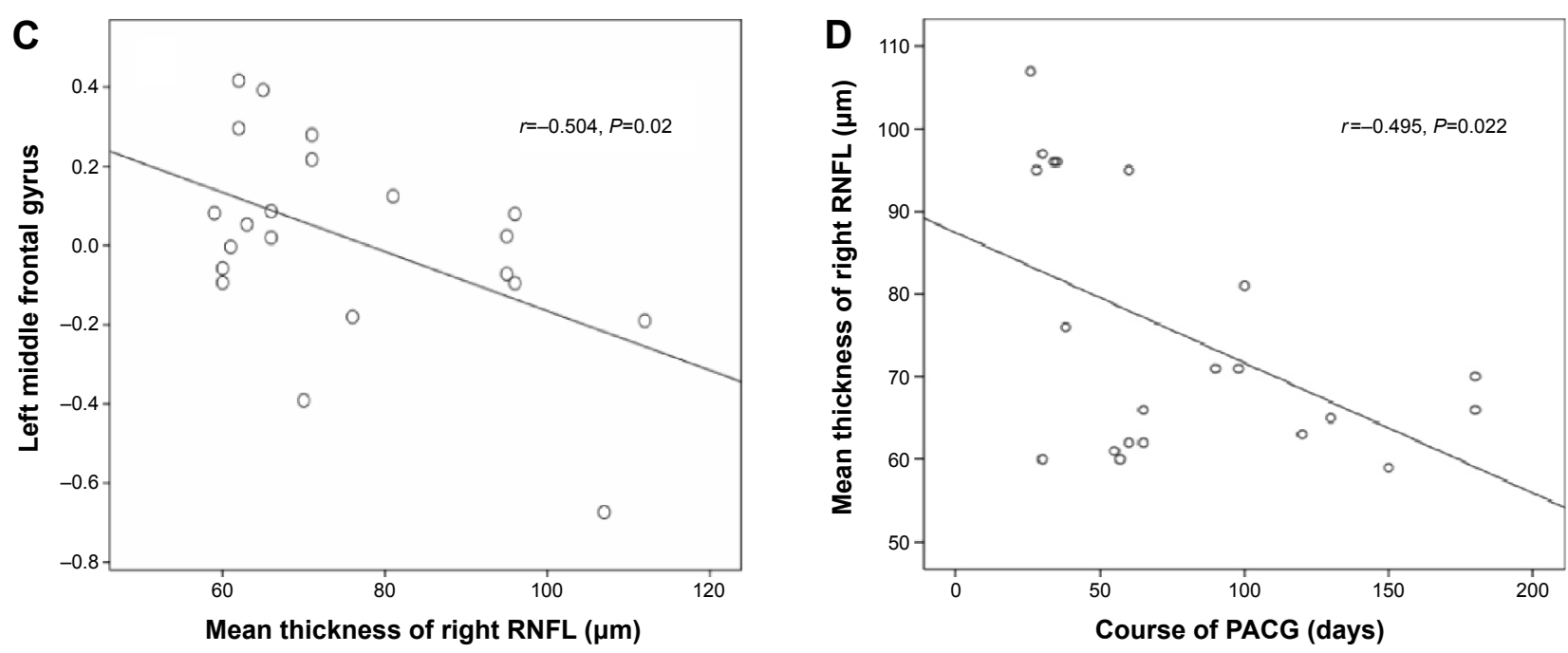

Figure 2 The correlations between the mean ALFF signal values of the different areas and the behavioral performances.

Notes: The stapeldiagram presents the mean ALFF signal value of the different brain regions $(\mathbf{A})$. The mean ALFF signal value of the right middle frontal gyrus showed a negative correlation with the left mean RNFLT $(B),(r=-0.487 ; P=0.033)$; the mean ALFF value of the left middle frontal gyrus showed a negative correlation with the right mean RNFLT (C), $(r=-0.504 ; P=0.02)$; and the right mean RNFLT showed a negative correlation with the course of the PACG (D), $(r=-0.495$; $P=0.022)$.

Abbreviations: ALFF, amplitude of low-frequency fluctuation; PACG, primary angle-closure glaucoma; HSs, healthy subjects; RNFLT, retinal nerve fiber layer thickness. 
Chen et $\mathrm{al}^{30}$ found that POAG had a smaller volume of gray matter in the superior frontal gyrus and inferior frontal gyrus compared with HSs. Song et $\mathrm{al}^{28}$ found that POAG patients showed significantly higher regional homogeneity areas in the bilateral medial frontal gyrus compared with HSs. Zikou et $\mathrm{al}^{31}$ found that POAG patients showed a significant decrease of FA value in the inferior fronto-occipital fasciculus and inferior frontal fasciculi, relative to HSs. In support of these findings, in the present study we found the lower ALFF areas in the bilateral middle frontal gyrus and bilateral superior frontal gyrus in PACG compared with HSs. Furthermore, the mean ALFF signal value of the middle frontal gyrus showed a negative correlation with the heterolateral mean RNFLT. These findings suggest that the PACG showed significant damage on the frontal gyrus, and the middle frontal gyrus could be useful for indexing the heterolateral RNFLT, which may be related to underlying neural mechanisms.

The precuneus is involved in the visuospatial imagery, episodic memory retrieval, self-processing, and consciousness. ${ }^{32,33}$ The posterior precuneus is primarily involved in the visuospatial functions. ${ }^{34,35}$ In a previous study, Song et $\mathrm{al}^{28}$ found decreased regional homogeneity in the bilateral precuneus in POAG. Chen et $\mathrm{al}^{30}$ found a significant increase in gray matter volume in the bilateral precuneus in POAG. Consistent with these findings, in the present study it was found that PACG showed lower ALFF in the right Precuneus. Therefore, we conclude that the decreased regional activity in the precuneus could reflect an impaired visual function.

\section{Conclusion}

In summary, our study demonstrated that PACG had abnormal regional spontaneous activities involved in the regional brain changes, which showed negative correlations with the RNFLT. These findings provide important information in order to understand the underlying neural mechanisms of PACG. Future studies are needed to recruit larger sample sizes to explore the differences of underlying functional mechanisms between the PACG with left and right eye disease.

\section{Acknowledgments}

This study was supported by the National Natural Science Foundation of China (81160118 and 81400372); Clinical Medicine Research Special-purpose Foundation of China (L2012052); Jiangxi Province Voyage Project (2014022);
Jiangxi Province Degree and Postgraduate Education Reform Project (2015); Science and Technology Platform Construction Project of Jiangxi Province (2013116); Youth Science Foundation of Jiangxi Province (20151BAB215016); Technology and Science Foundation of Jiangxi Province (20151BBG70223); Jiangxi Province Education Department Scientific Research Foundation (GJJ14170); and Health Development Planning Commission Science Foundation of Jiangxi Province (20155154).

\section{Disclosure}

This was not an industry supported study. The authors report no conflicts of interest in this work.

\section{References}

1. Quigley HA, Broman AT. The number of people with glaucoma worldwide in 2010 and 2020. Br J Ophthalmol. 2006;90(3):262-267.

2. Tarongoy P, Ho CL, Walton DS. Angle-closure glaucoma: the role of the lens in the pathogenesis, prevention, and treatment. Surv Ophthalmol. 2009;54(2):211-225.

3. Crawford ML, Harwerth RS, Smith EL 3rd, Mills S, Ewing B. Experimental glaucoma in primates: changes in cytochrome oxidase blobs in V1 cortex. Invest Ophthalmol Vis Sci. 2001;42(2):358-364.

4. Gupta N, Greenberg G, de Tilly LN, Gray B, Polemidiotis M, Yücel YH. Atrophy of the lateral geniculate nucleus in human glaucoma detected by magnetic resonance imaging. Br J Ophthalmol. 2009;93(1): $56-60$.

5. Gupta N, Ang LC, Noël de Tilly L, Bidaisee L, Yücel YH. Human glaucoma and neural degeneration in intracranial optic nerve, lateral geniculate nucleus, and visual cortex. Br J Ophthalmol. 2006;90(6): 674-678.

6. Gupta N, Yücel YH. What changes can we expect in the brain of glaucoma patients? Surv Ophthalmol. 2007;52(Suppl 2):S122-S126.

7. Engelhorn T, Michelson G, Waerntges S, Struffert T, Haider S, Doerfler A. Diffusion tensor imaging detects rarefaction of optic radiation in glaucoma patients. Acad Radiol. 2010;18(6):764-769.

8. Dai H, Yin D, Hu C, et al. Whole-brain voxel-based analysis of diffusion tensor MRI parameters in patients with primary open angle glaucoma and correlation with clinical glaucoma stage. Neuroradiology. 2013; 55(2):233-243.

9. Lee JY, Jeong HJ, Lee JH, et al. An investigation of lateral geniculate nucleus volume in patients with primary open-angle glaucoma using 7 tesla magnetic resonance imaging. Invest Ophthalmol Vis Sci. 2014:55(6):3468-3476.

10. Chen Z, Wang J, Lin F, Dai H, Mu K, Zhang H. Correlation between lateral geniculate nucleus atrophy and damage to the optic disc in glaucoma. J Neuroradiol. 2013;40(4):281-287.

11. Duncan RO, Sample PA, Bowd C, Weinreb RN, Zangwill LM. Arterial spin labeling fMRI measurements of decreased blood flow in primary visual cortex correlates with decreased visual function in human glaucoma. Vision Res. 2012;60:51-60.

12. Dai XJ, Liu CL, Zhou RL, et al. Long-term sleep deprivation decreases the default spontaneous activity and connectivity pattern in healthy male subjects: a resting-state fMRI study. Neuropsychiatr Dis Treat. 2015; 11:761-772.

13. Zuo XN, Di Martino A, Kelly C, et al. The oscillating brain: complex and reliable. Neuroimage. 2010;49(2):1432-1445.

14. Wang Z, Zhang Z, Liao W, et al. Frequency-dependent amplitude alterations of resting-state spontaneous fluctuations in idiopathic generalized epilepsy. Epilepsy Res. 2014;108(5):853-860. 
15. Kwak Y, Peltier SJ, Bohnen NI, Müller ML, Dayalu P, Seidler RD. L-DOPA changes spontaneous low-frequency BOLD signal oscillations in Parkinson's disease: a resting state fMRI study. Front Syst Neurosci. 2012;6:52

16. Li HJ, Dai XJ, Gong HH, Nie X, Zhang W, Peng DC. Aberrant spontaneous low-frequency brain activity in male patients with severe obstructive sleep apnea revealed by resting-state functional MRI. Neuropsychiatr Dis Treat. 2015;11:207-214.

17. Satterthwaite TD, Elliott MA, Gerraty RT, et al. An improved framework for confound regression and filtering for control of motion artifact in the preprocessing of resting-state functional connectivity data. Neuroimage. 2013;64:240-256.

18. Yan CG, Cheung B, Kelly C, et al. A comprehensive assessment of regional variation in the impact of head micromovements on functional connectomics. Neuroimage. 2013;76:183-201.

19. Fox MD, Snyder AZ, Vincent JL, Corbetta M, Van Essen DC, Raichle ME. The human brain is intrinsically organized into dynamic, anticorrelated functional networks. Proc Natl Acad Sci U S A. 2005;102 (27):9673-9678.

20. Dai XJ, Peng DC, Gong HH, et al. Altered intrinsic regional brain spontaneous activity and subjective sleep quality in patients with chronic primary insomnia: a resting-state fMRI study. Neuropsychiatr Dis Treat. 2014;10:2163-2175.

21. Saad ZS, Gotts SJ, Murphy K, et al. Trouble at rest: how correlation patterns and group differences become distorted after global signal regression. Brain Connect. 2012;2(1):25-32.

22. Zang YF, He Y, Zhu CZ, et al. Altered baseline brain activity in children with ADHD revealed by resting-state functional MRI. Brain Dev. 2007; 29(2):83-91.

23. van den Heuvel MP, Hulshoff Pol HE. Exploring the brain network: a review on resting-state fMRI functional connectivity. Eur Neuropsychopharmacol. 2010;20(8):519-534.

24. Peng DC, Dai XJ, Gong HH, Li HJ, Nie X, Zhang W. Altered intrinsic regional brain activity in male patients with severe obstructive sleep apnoea: A resting-state fMRI study. Neuropsychiatr Dis Treat. 2014;10: 1819-1826.
25. Dai XJ, Gong HH, Wang YX, et al. Gender differences in brain regional homogeneity of healthy subjects after normal sleep and after sleep deprivation: a resting-state fMRI study. Sleep Med. 2012;13(6):720-727.

26. Gao L, Bai LJ, Zhang YC, Dai XJ, Netra R, Min YJ, et al. Frequencydependent changes of local resting oscillations in sleep-deprived brain. PLoS One. 2015;10(3):e0120323.

27. Binkofski F, Fink GR, Geyer S, et al. Neural activity in human primary motor cortex areas $4 \mathrm{a}$ and $4 \mathrm{p}$ is modulated differentially by attention to action. J Neurophysiol. 2002;88(1):514-519.

28. Song Y, Mu K, Wang J, et al. Altered spontaneous brain activity in primary open angle glaucoma: a resting-state functional magnetic resonance imaging study. PLoS One. 2014;9(2):e89493.

29. Dai H, Morelli JN, Ai F, et al. Resting-state functional MRI: functional connectivity analysis of the visual cortex in primary open-angle glaucoma patients. Hum Brain Mapp. 2013;34(10):2455-2463.

30. Chen WW, Wang N, Cai S, et al. Structural brain abnormalities in patients with primary open-angle glaucoma: a study with 3T MR imaging. Invest Ophthalmol Vis Sci. 2013;54(1):545-554.

31. Zikou AK, Kitsos G, Tzarouchia LC, et al. Voxel-based morphometry and diffusion tensor imaging of the optic pathway in primary open-angle glaucoma: a preliminary study. AJNR Am J Neuroradiol. 2012;33(1): $128-134$.

32. Davidson RJ, Abercrombie H, Nitschke JB, Putnam K. Regional brain function, emotion and disorders of emotion. Curr Opin Neurobiol. 1999;9(2): 228-234.

33. Cavanna AE, Trimble MR. The precuneus: a review of its functional anatomy and behavioural correlates. Brain. 2006;129(Pt 3):564-583.

34. Colby CL, Gattass R, Olson CR, Gross CG. Topographical organization of cortical afferents to extrastriate visual area PO in the macaque: a dual tracer study. J Comp Neurol. 1988;269(3):392-413.

35. Wang K, Jiang T, Yu C, et al. Spontaneous activity associated with primary visual cortex: a resting-state FMRI study. Cereb Cortex. 2008;18(3) 697-704.
Neuropsychiatric Disease and Treatment

\section{Publish your work in this journal}

Neuropsychiatric Disease and Treatment is an international, peerreviewed journal of clinical therapeutics and pharmacology focusing on concise rapid reporting of clinical or pre-clinical studies on a range of neuropsychiatric and neurological disorders. This journal is indexed on PubMed Central, the 'PsycINFO' database and CAS,

\section{Dovepress}

and is the official journal of The International Neuropsychiatric Association (INA). The manuscript management system is completely online and includes a very quick and fair peer-review system, which is all easy to use. Visit http://www.dovepress.com/testimonials.php to read real quotes from published authors. 